\title{
Bag of Tricks for Efficient Text Classification
}

\author{
Armand Joulin Edouard Grave Piotr Bojanowski Tomas Mikolov \\ Facebook AI Research \\ \{ajoulin, egrave, bojanowski, tmikolov\}afb.com
}

\begin{abstract}
This paper explores a simple and efficient baseline for text classification. Our experiments show that our fast text classifier fastText is often on par with deep learning classifiers in terms of accuracy, and many orders of magnitude faster for training and evaluation. We can train fast Text on more than one billion words in less than ten minutes using a standard multicore CPU, and classify half a million sentences among $312 \mathrm{~K}$ classes in less than a minute.
\end{abstract}

\section{Introduction}

Text classification is an important task in Natural Language Processing with many applications, such as web search, information retrieval, ranking and document classification (Deerwester et al., 1990; Pang and Lee, 2008). Recently, models based on neural networks have become increasingly popular (Kim, 2014; Zhang and LeCun, 2015; Conneau et al., 2016). While these models achieve very good performance in practice, they tend to be relatively slow both at train and test time, limiting their use on very large datasets.

Meanwhile, linear classifiers are often considered as strong baselines for text classification problems (Joachims, 1998; McCallum and Nigam, 1998; Fan et al., 2008). Despite their simplicity, they often obtain state-of-the-art performances if the right features are used (Wang and Manning, 2012). They also have the potential to scale to very large corpus (Agarwal et al., 2014).

In this work, we explore ways to scale these baselines to very large corpus with a large output space, in the context of text classification. Inspired by the recent work in efficient word representation learning (Mikolov et al., 2013; Levy et al., 2015), we show that linear models with a rank constraint and a fast loss approximation can train on a billion words within ten minutes, while achieving performance on par with the state-of-the-art. We evaluate the quality of our approach fast Text ${ }^{1}$ on two different tasks, namely tag prediction and sentiment analysis.

\section{Model architecture}

A simple and efficient baseline for sentence classification is to represent sentences as bag of words (BoW) and train a linear classifier, e.g., a logistic regression or an SVM (Joachims, 1998; Fan et al., 2008). However, linear classifiers do not share parameters among features and classes. This possibly limits their generalization in the context of large output space where some classes have very few examples. Common solutions to this problem are to factorize the linear classifier into low rank matrices (Schütze, 1992; Mikolov et al., 2013) or to use multilayer neural networks (Collobert and Weston, 2008; Zhang et al., 2015).

Figure 1 shows a simple linear model with rank constraint. The first weight matrix $A$ is a look-up table over the words. The word representations are then averaged into a text representation, which is in turn fed to a linear classifier. The text representation is an hidden variable which can be potentially be reused. This architecture is similar to the cbow model of Mikolov et al. (2013), where the middle word is replaced by a label. We use the softmax function $f$ to compute the probability distribution over the predefined classes. For a set of $N$ documents, this leads to minimizing the negative log-likelihood over the classes:

$$
-\frac{1}{N} \sum_{n=1}^{N} y_{n} \log \left(f\left(B A x_{n}\right)\right),
$$

\footnotetext{
${ }^{1}$ https://github.com/facebookresearch/ fast Text
} 


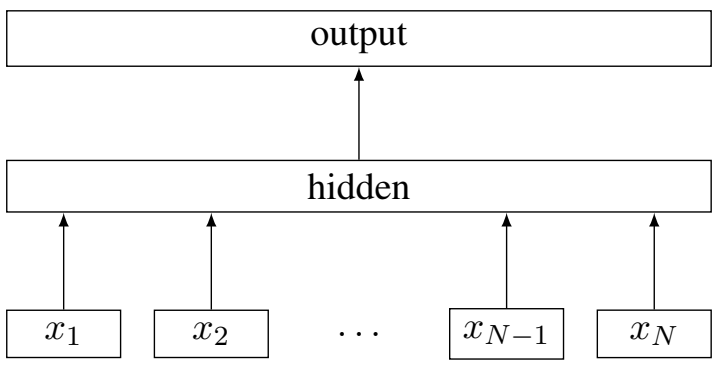

Figure 1: Model architecture of fast Text for a sentence with $N$ ngram features $x_{1}, \ldots, x_{N}$. The features are embedded and averaged to form the hidden variable.

where $x_{n}$ is the normalized bag of features of the $n$-th document, $y_{n}$ the label, $A$ and $B$ the weight matrices. This model is trained asynchronously on multiple CPUs using stochastic gradient descent and a linearly decaying learning rate.

\subsection{Hierarchical softmax}

When the number of classes is large, computing the linear classifier is computationally expensive. More precisely, the computational complexity is $O(k h)$ where $k$ is the number of classes and $h$ the dimension of the text representation. In order to improve our running time, we use a hierarchical softmax (Goodman, 2001) based on the Huffman coding tree (Mikolov et al., 2013).

During training, the computational complexity drops to $O\left(h \log _{2}(k)\right)$.

The hierarchical softmax is also advantageous at test time when searching for the most likely class. Each node is associated with a probability that is the probability of the path from the root to that node. If the node is at depth $l+1$ with parents $n_{1}, \ldots, n_{l}$, its probability is

$$
P\left(n_{l+1}\right)=\prod_{i=1}^{l} P\left(n_{i}\right)
$$

This means that the probability of a node is always lower than the one of its parent. Exploring the tree with a depth first search and tracking the maximum probability among the leaves allows us to discard any branch associated with a small probability. In practice, we observe a reduction of the complexity to $O\left(h \log _{2}(k)\right)$ at test time. This approach is further extended to compute the $T$-top targets at the cost of $O(\log (T))$, using a binary heap.

\section{$2.2 \quad \mathrm{~N}$-gram features}

Bag of words is invariant to word order but taking explicitly this order into account is often computationally very expensive. Instead, we use a bag of n-grams as additional features to capture some partial information about the local word order. This is very efficient in practice while achieving comparable results to methods that explicitly use the order (Wang and Manning, 2012).

We maintain a fast and memory efficient mapping of the n-grams by using the hashing trick (Weinberger et al., 2009) with the same hashing function as in Mikolov et al. (2011) and 10M bins if we only used bigrams, and 100M otherwise.

\section{Experiments}

We evaluate fast Text on two different tasks. First, we compare it to existing text classifers on the problem of sentiment analysis. Then, we evaluate its capacity to scale to large output space on a tag prediction dataset. Note that our model could be implemented with the Vowpal Wabbit library, ${ }^{2}$ but we observe in practice, that our tailored implementation is at least $2-5 \times$ faster.

\subsection{Sentiment analysis}

Datasets and baselines. We employ the same 8 datasets and evaluation protocol of Zhang et al. (2015). We report the n-grams and TFIDF baselines from Zhang et al. (2015), as well as the character level convolutional model (charCNN) of Zhang and LeCun (2015), the character based convolution recurrent network (charCRNN) of (Xiao and Cho, 2016) and the very deep convolutional network (VDCNN) of Conneau et al. (2016). We also compare to Tang et al. (2015) following their evaluation protocol. We report their main baselines as well as their two approaches based on recurrent networks (ConvGRNN and LSTM-GRNN).

Results. We present the results in Figure 1. We use 10 hidden units and run fast Text for 5 epochs with a learning rate selected on a validation set from $\{0.05,0.1,0.25,0.5\}$. On this task, adding bigram information improves the performance by $1-4 \%$. Overall our accuracy is slightly better than char-CNN and char-CRNN and, a bit

\footnotetext{
${ }^{2}$ Using the options --nn, --ngrams and --log_multi
} 


\begin{tabular}{lcccccccc}
\hline Model & AG & Sogou & DBP & Yelp P. & Yelp F. & Yah. A. & Amz. F. & Amz. P. \\
\hline BoW (Zhang et al., 2015) & 88.8 & 92.9 & 96.6 & 92.2 & 58.0 & 68.9 & 54.6 & 90.4 \\
ngrams (Zhang et al., 2015) & 92.0 & 97.1 & 98.6 & 95.6 & 56.3 & 68.5 & 54.3 & 92.0 \\
ngrams TFIDF (Zhang et al., 2015) & 92.4 & 97.2 & 98.7 & 95.4 & 54.8 & 68.5 & 52.4 & 91.5 \\
char-CNN (Zhang and LeCun, 2015) & 87.2 & 95.1 & 98.3 & 94.7 & 62.0 & 71.2 & 59.5 & 94.5 \\
char-CRNN (Xiao and Cho, 2016) & 91.4 & 95.2 & 98.6 & 94.5 & 61.8 & 71.7 & 59.2 & 94.1 \\
VDCNN (Conneau et al., 2016) & 91.3 & 96.8 & 98.7 & 95.7 & 64.7 & 73.4 & 63.0 & 95.7 \\
\hline fast Text, $h=10$ & 91.5 & 93.9 & 98.1 & 93.8 & 60.4 & 72.0 & 55.8 & 91.2 \\
fast Text, $h=10$, bigram & 92.5 & 96.8 & 98.6 & 95.7 & 63.9 & 72.3 & 60.2 & 94.6 \\
\hline
\end{tabular}

Table 1: Test accuracy [\%] on sentiment datasets. Fast Text has been run with the same parameters for all the datasets. It has 10 hidden units and we evaluate it with and without bigrams. For char-CNN, we show the best reported numbers without data augmentation.

\begin{tabular}{|c|c|c|c|c|c|c|}
\hline & \multicolumn{2}{|c|}{ Zhang and LeCun (2015) } & \multicolumn{3}{|c|}{ Conneau et al. (2016) } & \multirow{2}{*}{$\begin{array}{c}\text { fast Text } \\
h=10 \text {, bigram }\end{array}$} \\
\hline & small char-CNN & big char-CNN & depth $=9$ & depth $=17$ & depth $=29$ & \\
\hline AG & $1 \mathrm{~h}$ & $3 \mathrm{~h}$ & $24 \mathrm{~m}$ & $37 \mathrm{~m}$ & $51 \mathrm{~m}$ & $1 \mathrm{~s}$ \\
\hline Sogou & - & - & $25 \mathrm{~m}$ & $41 \mathrm{~m}$ & $56 \mathrm{~m}$ & $7 \mathrm{~s}$ \\
\hline DBpedia & $2 \mathrm{~h}$ & $5 \mathrm{~h}$ & $27 \mathrm{~m}$ & $44 m$ & $1 \mathrm{~h}$ & $2 s$ \\
\hline Yelp P. & - & - & $28 \mathrm{~m}$ & $43 m$ & 1h09 & $3 \mathrm{~s}$ \\
\hline Yelp F. & - & - & $29 m$ & $45 \mathrm{~m}$ & 1h12 & $4 \mathrm{~s}$ \\
\hline Yah. A. & $8 \mathrm{~h}$ & $1 d$ & $1 \mathrm{~h}$ & $1 \mathrm{~h} 33$ & $2 \mathrm{~h}$ & $5 s$ \\
\hline Amz. F. & $2 d$ & $5 d$ & $2 \mathrm{~h} 45$ & $4 \mathrm{~h} 20$ & $7 \mathrm{~h}$ & $9 \mathrm{~s}$ \\
\hline Amz. P. & $2 d$ & $5 \mathrm{~d}$ & $2 \mathrm{~h} 45$ & $4 \mathrm{~h} 25$ & $7 \mathrm{~h}$ & $10 \mathrm{~s}$ \\
\hline
\end{tabular}

Table 2: Training time for a single epoch on sentiment analysis datasets compared to char-CNN and VDCNN.

worse than VDCNN. Note that we can increase the accuracy slightly by using more n-grams, for example with trigrams, the performance on Sogou goes up to $97.1 \%$. Finally, Figure 3 shows that our method is competitive with the methods presented in Tang et al. (2015). We tune the hyperparameters on the validation set and observe that using n-grams up to 5 leads to the best performance. Unlike Tang et al. (2015), fast Text does not use pre-trained word embeddings, which can be explained the $1 \%$ difference in accuracy.

\begin{tabular}{lcccc}
\hline Model & Yelp'13 & Yelp'14 & Yelp'15 & IMDB \\
\hline SVM+TF & 59.8 & 61.8 & 62.4 & 40.5 \\
CNN & 59.7 & 61.0 & 61.5 & 37.5 \\
Conv-GRNN & 63.7 & 65.5 & 66.0 & 42.5 \\
LSTM-GRNN & 65.1 & 67.1 & 67.6 & 45.3 \\
\hline fastText & 64.2 & 66.2 & 66.6 & 45.2 \\
\hline
\end{tabular}

Table 3: Comparision with Tang et al. (2015). The hyper-parameters are chosen on the validation set. We report the test accuracy.

Training time. Both char-CNN and VDCNN are trained on a NVIDIA Tesla K40 GPU, while our models are trained on a CPU using 20 threads. Table 2 shows that methods us- ing convolutions are several orders of magnitude slower than fastText. While it is possible to have a $10 \times$ speed up for char-CNN by using more recent CUDA implementations of convolutions, fast Text takes less than a minute to train on these datasets. The GRNNs method of Tang et al. (2015) takes around 12 hours per epoch on CPU with a single thread. Our speed-up compared to neural network based methods increases with the size of the dataset, going up to at least a $15,000 \times$ speed-up.

\subsection{Tag prediction}

Dataset and baselines. To test scalability of our approach, further evaluation is carried on the YFCC100M dataset (Thomee et al., 2016) which consists of almost 100M images with captions, titles and tags. We focus on predicting the tags according to the title and caption (we do not use the images). We remove the words and tags occurring less than 100 times and split the data into a train, validation and test set. The train set contains 91,188,648 examples (1.5B tokens). The validation has 930,497 examples and the test set 543,424 . The vocabulary size is 297,141 and there are 312,116 unique tags. We will release a script that recreates this dataset so that our num- 


\begin{tabular}{lll}
\hline Input & Prediction & Tags \\
\hline $\begin{array}{l}\text { taiyoucon 2011 digitals: individuals digital pho- } \\
\text { tos from the anime convention taiyoucon 2011 in } \\
\text { mesa, arizona. if you know the model and/or the } \\
\text { character, please comment. }\end{array}$ & \#cosplay & $\begin{array}{l}\text { \#24mm \#anime \#animeconvention } \\
\text { \#arizona \#canon \#con \#convention } \\
\text { \#cos \#cosplay \#costume \#mesa \#play } \\
\text { \#taiyou \#taiyoucon }\end{array}$ \\
\hline $\begin{array}{l}2012 \text { twin cities pride 2012 twin cities pride pa- } \\
\text { rade }\end{array}$ & \#minneapolis & $\begin{array}{l}\text { \#2012twincitiesprideparade \#min- } \\
\text { neapolis \#mn \#usa }\end{array}$ \\
\hline beagle enjoys the snowfall & \#snow & $\begin{array}{l}\text { \#2007 \#beagle \#hillsboro \#january } \\
\text { \#maddison \#maddy \#oregon \#snow }\end{array}$ \\
\hline christmas & \#christmas & \#cameraphone \#mobile \\
\hline euclid avenue & \#newyorkcity & \#cleveland \#euclidavenue \\
\hline
\end{tabular}

Table 4: Examples from the validation set of YFCC100M dataset obtained with fastText with 200 hidden units and bigrams. We show a few correct and incorrect tag predictions.

\begin{tabular}{lccc}
\hline \multirow{2}{*}{ Model } & \multirow{2}{*}{ prec@ 1 } & \multicolumn{2}{c}{ Running time } \\
\cline { 3 - 4 } & & Train & Test \\
\hline Freq. baseline & 2.2 & - & - \\
Tagspace, $h=50$ & 30.1 & $3 \mathrm{~h} 8$ & $6 \mathrm{~h}$ \\
Tagspace, $h=200$ & 35.6 & $5 \mathrm{~h} 32$ & $15 \mathrm{~h}$ \\
\hline fastText, $h=50$ & 31.2 & $6 \mathrm{~m} 40$ & $48 \mathrm{~s}$ \\
fastText, $h=50$, bigram & 36.7 & $7 \mathrm{~m} 47$ & $50 \mathrm{~s}$ \\
fastText, $h=200$ & 41.1 & $10 \mathrm{~m} 34$ & $1 \mathrm{~m} 29$ \\
fastText, $h=200$, bigram & 46.1 & $13 \mathrm{~m} 38$ & $1 \mathrm{~m} 37$ \\
\hline
\end{tabular}

Table 5: Prec@1 on the test set for tag prediction on YFCC100M. We also report the training time and test time. Test time is reported for a single thread, while training uses 20 threads for both models.

bers could be reproduced. We report precision at 1 .

We consider a frequency-based baseline which predicts the most frequent tag. We also compare with Tagspace (Weston et al., 2014), which is a tag prediction model similar to ours, but based on the Wsabie model of Weston et al. (2011). While the Tagspace model is described using convolutions, we consider the linear version, which achieves comparable performance but is much faster.

Results and training time. Table 5 presents a comparison of fast Text and the baselines. We run fastText for 5 epochs and compare it to Tagspace for two sizes of the hidden layer, i.e., 50 and 200. Both models achieve a similar performance with a small hidden layer, but adding bigrams gives us a significant boost in accuracy. At test time, Tagspace needs to compute the scores for all the classes which makes it relatively slow, while our fast inference gives a sig- nificant speed-up when the number of classes is large (more than $300 \mathrm{~K}$ here). Overall, we are more than an order of magnitude faster to obtain model with a better quality. The speedup of the test phase is even more significant (a $600 \times$ speedup). Table 4 shows some qualitative examples.

\section{Discussion and conclusion}

In this work, we propose a simple baseline method for text classification. Unlike unsupervisedly trained word vectors from word2vec, our word features can be averaged together to form good sentence representations. In several tasks, fast Text obtains performance on par with recently proposed methods inspired by deep learning, while being much faster. Although deep neural networks have in theory much higher representational power than shallow models, it is not clear if simple text classification problems such as sentiment analysis are the right ones to evaluate them. We will publish our code so that the research community can easily build on top of our work.

Acknowledgement. We thank Gabriel Synnaeve, Hervé Gégou, Jason Weston and Léon Bottou for their help and comments. We also thank Alexis Conneau, Duyu Tang and Zichao Zhang for providing us with information about their methods.

\section{References}

Alekh Agarwal, Olivier Chapelle, Miroslav Dudík, and John Langford. 2014. A reliable effective terascale linear learning system. Journal of Machine Learning Research, 15(Mar):1111-1133.

Ronan Collobert and Jason Weston. 2008. A unified architecture for natural language processing: 
Deep neural networks with multitask learning. In Proceedings of the 25th International Conference on Machine Learning, ICML '08, pages 160-167, Helsinki, Finland. ACM.

Alexis Conneau, Holger Schwenk, Loïc Barrault, and Yann Lecun. 2016. Very deep convolutional networks for natural language processing. arXiv preprint arXiv:1606.01781.

Scott Deerwester, Susan T. Dumais, George W. Furnas, Thomas K. Landauer, and Richard Harshman. 1990. Indexing by latent semantic analysis. Journal of the American Society for Information Science, 41(6):391-407.

Rong-En Fan, Kai-Wei Chang, Cho-Jui Hsieh, XiangRui Wang, and Chih-Jen Lin. 2008. Liblinear: A library for large linear classification. Journal of Machine Learning Research, 9(Aug):1871-1874.

Joshua Goodman. 2001. Classes for fast maximum entropy training. In Proceedings of the International Conference on Acoustics, Speech, and Signal Processing, volume 1, pages 561-564, Salt Lake City, USA. IEEE.

Thorsten Joachims. 1998. Text categorization with support vector machines: Learning with many relevant features. In Claire Nédellec and Céline Rouveirol, editors, 10th European Conference on Machine Learning, pages 137-142, Chemnitz, Germany. Springer Berlin Heidelberg.

Yoon Kim. 2014. Convolutional neural networks for sentence classification. In Proceedings of the 2014 Conference on Empirical Methods in Natural Language Processing (EMNLP), pages 17461751, Doha, Qatar, October. Association for Computational Linguistics.

Omer Levy, Yoav Goldberg, and Ido Dagan. 2015. Improving distributional similarity with lessons learned from word embeddings. Transactions of the Association for Computational Linguistics, 3:211-225.

Andrew McCallum and Kamal Nigam. 1998. A comparison of event models for naive bayes text classification. In AAAI workshop on learning for text categorization, pages 41-48, Madison, USA.

Tomáš Mikolov, Anoop Deoras, Daniel Povey, Lukáš Burget, and Jan Černockỳ. 2011. Strategies for training large scale neural network language models. In Workshop on Automatic Speech Recognition Understanding, pages 196-201, Waikoloa, USA. IEEE.

Tomáš Mikolov, Kai Chen, Greg Corrado, and Jeffrey Dean. 2013. Efficient estimation of word representations in vector space. In 1st International Conference on Learning Representations (ICLR), Scottsdale, USA.

Bo Pang and Lillian Lee. 2008. Opinion mining and sentiment analysis. Foundations and Trends in Information Retrieval, 2(1-2):1-135, January.
H. Schütze. 1992. Dimensions of meaning. In Proceedings of the 1992 ACM/IEEE Conference on Supercomputing, Supercomputing '92, pages 787-796, Los Alamitos, CA, USA. IEEE Computer Society Press.

Duyu Tang, Bing Qin, and Ting Liu. 2015. Document modeling with gated recurrent neural network for sentiment classification. In Proceedings of the 2015 Conference on Empirical Methods in Natural Language Processing, pages 1422-1432, Lisbon, Portugal, September. Association for Computational Linguistics.

Bart Thomee, David A. Shamma, Gerald Friedland, Benjamin Elizalde, Karl Ni, Douglas Poland, Damian Borth, and Li-Jia Li. 2016. YFCC100M: The new data in multimedia research. Communications of the ACM, 59(2):64-73.

Sida Wang and Christopher Manning. 2012. Baselines and bigrams: Simple, good sentiment and topic classification. In Proceedings of the 50th Annual Meeting of the Association for Computational Linguistics (Volume 2: Short Papers), pages 90-94, Jeju Island, Korea, July. Association for Computational Linguistics.

Kilian Weinberger, Anirban Dasgupta, John Langford, Alex Smola, and Josh Attenberg. 2009. Feature hashing for large scale multitask learning. In Proceedings of the 26th Annual International Conference on Machine Learning, ICML '09, pages 11131120, New York, NY, USA. ACM.

Jason Weston, Samy Bengio, and Nicolas Usunier. 2011. Wsabie: Scaling up to large vocabulary image annotation. In Proceedings of the Twenty-Second International Joint Conference on Artificial Intelligence - Volume Volume Three, IJCAI'11, pages 2764-2770. AAAI Press.

Jason Weston, Sumit Chopra, and Keith Adams. 2014. \#tagspace: Semantic embeddings from hashtags. In Proceedings of the 2014 Conference on Empirical Methods in Natural Language Processing (EMNLP), pages 1822-1827, Doha, Qatar, October. Association for Computational Linguistics.

Yijun Xiao and Kyunghyun Cho. 2016. Efficient character-level document classification by combining convolution and recurrent layers. arXiv preprint arXiv:1602.00367.

Xiang Zhang and Yann LeCun. 2015. Text understanding from scratch. arXiv preprint arXiv:1502.01710.

Xiang Zhang, Junbo Zhao, and Yann LeCun. 2015. Character-level convolutional networks for text classification. In Advances in Neural Information Processing Systems 28, pages 649-657, Montreal, Canada. 